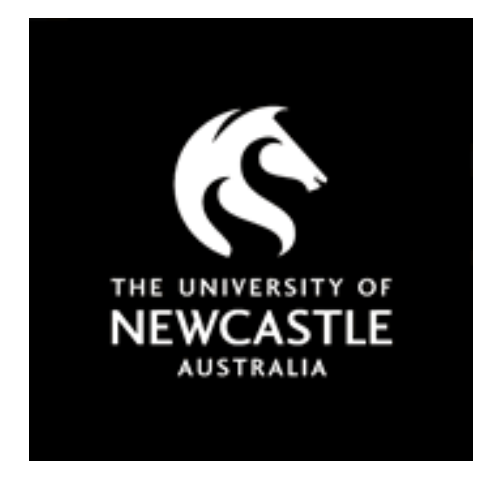

NOVA

University of Newcastle Research Online

nova.newcastle.edu.au

Keast, V. J., Myles, T. A., Shahcheraghi, N., Cortie, M. B. Springer. "Corrosion processes of triangular silver nanoparticles compared to bulk silver", Published in Journal of Nanoparticle Research Vol. 18, Issue 2, no. 45. (2016)

Available from: http://dx.doi.org/10.1007/s11051-016-3354-9

The original publication is available at www.springerlink.com.

Accessed from: http://hdl.handle.net/1959.13/1346037 


\section{Corrosion processes of triangular silver nanoparticles compared to bulk silver}

\section{J. Keast ${ }^{\dagger *}$, T. A. Myles ${ }^{\dagger}$, N. Shahcheraghi ${ }^{\ddagger}$ and M. B. Cortie ${ }^{\ddagger}$}

${ }^{\dagger}$ School of Mathematical and Physical Sciences, University of Newcastle, Callaghan NSW 2308,

Australia.

${ }^{\ddagger}$ Institute for Nanoscale Technology, University of Technology Sydney, Broadway, NSW 2007, Australia.

Corresponding Author

Assoc. Prof. Vicki J. Keast

School of Mathematical and Physical Sciences

The University of Newcastle

Callaghan NSW 2308

Australia

Ph: + 61249216653

Fax: + 61249216907

email: vicki.keast@newcastle.edu.au

\section{Acknowledgements}

This research was supported under Australian Research Council’s Discovery Projects funding scheme (Project Number DP120102545). The authors acknowledge use of the facilities at the Monash Centre for Electron Microscopy and the assistance of Laure Bourgeois with sample preparation. 


\begin{abstract}
Excessive corrosion of silver nanoparticles is a significant impediment to their use in a variety of potential applications in the biosensing, plasmonic and antimicrobial fields. Here we examine the environmental degradation of triangular silver nanoparticles (AgNP) in laboratory air. In the early stages of corrosion, transmission electron microscopy shows that dissolution of the single-crystal, triangular, AgNP (side lengths 50-120 nm) is observed with the accompanying formation of smaller, polycrystalline Ag particles nearby. The new particles are then observed to corrode to $\mathrm{Ag}_{2} \mathrm{~S}$ and after 21 days nearly full corrosion has occurred, but some with minor Ag inclusions remaining. In contrast, a bulk Ag sheet, studied in cross-section, showed an adherent corrosion layer of only around 20-50 nm in thickness after over a decade of being exposed to ambient air. The results have implications for antibacterial properties and ecotoxicology of AgNP during corrosion as the dissolution and reformation of Ag particles during corrosion will likely be accompanied by the release of $\mathrm{Ag}^{+}$ions.
\end{abstract}

\title{
Keywords
}

Nanoparticle, plasmonics, corrosion, silver 


\section{Introduction}

There is considerable interest in the properties of silver nanoparticles (AgNP). This is motivated by their current and potential applications across a diverse range of health and engineering fields. Use of colloidal Ag for health related applications has a long history (Alexander 2009) but, more recently, AgNP have found applications in wound and burn dressings, in medical instruments and implants, in fabrics for odour reduction, and in wide array of consumer products and cosmetics (Chernousova and Epple 2013; Marambio-Jones and Hoek 2010; Reidy et al. 2013). AgNP also have potential for plasmonic applications as they have an exceptionally strong plasmon response (Pastoriza-Santos and Liz-Marzan 2008; Rycenga et al. 2011). This response is exploited for surface enhanced Raman spectroscopy (SERS), an analytical technique of great sensitivity (Fan and Brolo 2009).

Triangular nanoplates of Ag are attractive for optical applications as they can be readily synthesized and display a number of well-developed dipole and multipole plasmon resonances (Fletcher et al. 2015; Keast et al. 2016; Yang et al. 2009). The multipolar resonances are excited at smaller particle sizes, relative to the situation for spheres or rods, due to the lower symmetry of the triangles. The wavelengths at which these resonances occur can be readily tuned by adjustment of their shape (Kelly et al. 2003). In addition, Ag nanotriangles in the tip-to-tip configuration (the so-called 'nanobowtie') present a powerful platform for developing SERS applications spectroscopy due to the strong electric field between the tips (Kinkhabwala et al. 2009). 
However, the potential of AgNP in practical plasmonic devices has not yet been fully realised due to their rapid corrosion in ambient air. The corrosion process of AgNP will also play an important role in their effectiveness in medical and antibacterial applications and may be of significance in resolving some of the inconsistencies reported in regard to antimicrobial action in real-world situations when compared to in vitro studies (Marx and Barillo 2014; McQueen et al. 2013; StormVersloot et al. 2010; Toy and Macera 2011; Walter et al. 2014). Additionally, an understanding of the corrosion of Ag is also necessary for the conservation and restoration of historical and cultural objects. From an environmental perspective, the growing use of AgNP in wide variety of medical, technical and consumer products gives rise to significant concerns about their ecotoxic effects upon their later release to the environment (Reidy et al. 2013). The nature, extent and time-frame of the corrosion process will be of critical importance for evaluating these environmental impacts.

A corrosion layer of $\mathrm{Ag}_{2} \mathrm{~S}$ forms on bulk $\mathrm{Ag}$ in normal laboratory air and does not form in a dry nitrogen environment. The formation of an $\mathrm{Ag}_{2} \mathrm{~S}$ corrosion layer requires the presence of both oxygen and water vapour and the growth rate depends on the environment (Bennett et al. 1969; Burge et al. 1969; Kleber et al. 2008; Pope et al. 1968). Atmospheric corrosion is principally by $\mathrm{H}_{2} \mathrm{~S}$ and OCS (carbonyl sulphide) (Franey et al. 1985) although corrosion by organic sulfur species has also been observed (Sinclair 1982). The corrosion has been observed to form initially as a discontinuous layer of spherical particles where the particles increase in size but not density as the corrosion proceeds (Bennett et al. 1969; Burge et al. 1969; Kleber et al. 2008; Pope et al. 1968). 
The antimicrobial properties of Ag are believed to be predominantly related to the release of $\mathrm{Ag}^{+}$ ions, which is enhanced by the relatively large surface area of AgNP when compared to the bulk form (Le Ouay and Sellacci 2015; Rizzello and Pompa 2014; Xiu et al. 2012). The sulfidation of AgNP has been observed to produce a dramatic reduction in the antibacterial effects (Reinsch et al. 2012) as the limited solubility of $\mathrm{Ag}_{2} \mathrm{~S}$ means the release of $\mathrm{Ag}^{+}$ions will be very slow once transformation has occurred. The $\mathrm{Ag}^{+}$ion release and antimicrobial effect is enhanced for AgNP with structural defects (Munusamy et al. 2015) and for flat triangular AgNPs (Lu et al. 2015; Pal et al. 2007). While corrosion is also enhanced at defects, it is slower on the $\{111\}$ planes that dominate the flat triangular Ag-NP structures and so further insight into the relationship between Ag-NP structure and corrosion is needed (Allpress and Sanders 1964; Miwa and Watanabe 1947; Philips 1962; Shiojiri et al. 1969).

The intentional and environmental sulfidation of AgNP has been extensively studied and a variety of effects have been observed (Andrieux-Ledier et al. 2013; Cao and Elsayed-Ali 2009; Chen et al. 2008; Chen et al. 2013a; Elechiguerra et al. 2005; Huang et al. 2014; Levard et al. 2012; Levard et al. 2011; Liu and Ma 2011; Lu et al. 2015; Mayousse et al. 2015; McMahon et al. 2005; Park et al. 2012; Thalman et al. 2014; Theodorou et al. 2015; Wang et al. 2011; Zeng et al. 2011). The corrosion product has been observed as an inhomogeneous $\mathrm{Ag}_{2} \mathrm{~S}$ shell around the particles, (Chen et al. 2013a; Chen et al. 2013b; Elechiguerra et al. 2005) or as the formation of separate, smaller $\mathrm{Ag}_{2} \mathrm{~S}$ particles on the surface or nearby (Chen et al. 2013a; Chen et al. 2013b; Elechiguerra et al. 2005; Lu et al. 2015; Wang et al. 2011). In instances of intentional sulfidation using solutions of high 
$\mathrm{Na}_{2} \mathrm{~S}$ concentrations, the formation of bridges between the particles (Levard et al. 2011) or conversion at the tips and corners of particles (Huang et al. 2014; Liu and Ma 2011; Zeng et al. 2011) is observed.

It has been proposed that there are two distinct processes that can occur: oxidative dissolution of Ag to $\mathrm{Ag}^{+}$followed by precipitation as $\mathrm{Ag}_{2} \mathrm{~S}$; or direct conversion to $\mathrm{Ag}_{2} \mathrm{~S}$. The oxidative dissolution route dominates at low S and the direct route at higher S concentrations.(Liu et al. 2011) The dissolution route has implications for antimicrobial activity because of the important role of the $\mathrm{Ag}^{+}$ ions in this process. In some cases, the formation of pure Ag secondary particles has been observed (Davidson et al. 2014; Glover et al. 2011; Theodorou et al. 2015) and this also occurred for bulk Ag objects (Glover et al. 2011).

It is clear that the chemical environment and atomic structure play a critical role in the corrosion process and that our understanding of the chemical transformation processes in AgNP, in particular, is incomplete. Furthermore, the observation of particulate-like reactions in the early stages of bulk Ag corrosion (Glover et al. 2011) may indicate that the corrosion processes in bulk Ag and AgNP have more in common than is commonly assumed. At the heart of both the corrosion process and the antimicrobial behaviour of $\mathrm{Ag}$ is the release of $\mathrm{Ag}^{+}$ions and their subsequent interactions with other species in the local environment. In the present work we address these questions by harnessing transmission electron microscopy (TEM) to examine the corrosion of bulk Ag and triangular AgNP in order to provide further insight into this complex process. 


\section{Experimental}

\section{Bulk Ag Sheet}

The bulk specimen was a $100 \mu$ m thick 99.999\% pure Ag sheet (Alfa Aesar). It had been previously used to prepare TEM specimens for another study and had since been stored in a variety of office and laboratory environments for over 10 years. As a consequence of handling and long-term storage it had developed an obvious corrosion layer typical of that observed on bulk Ag objects (see the photograph of Fig. 1a). A section from the boxed area indicated was removed and sandwiched between pieces of single crystal Si wafer. A cross-sectional TEM specimen was then prepared by mechanical polishing, dimpling and ion-beam milling. A nearby area was removed for observation in the scanning electron microscope (SEM) in order to determine whether the regions displaying the different colors of the corrosion layer showed any microstructural differences.

\section{Flat Triangular AgNP}

Samples of flat triangular AgNP were prepared by reacting $\mathrm{AgNO}_{3}$ and with $\mathrm{NaBH}_{4}$, using polyvinylpyrrolidone as a protective surfactant, to produce nanoparticle nuclei. These were then grown in a solution containing trisodium citrate and $\mathrm{H}_{2} \mathrm{O}_{2}$. (Tsuji et al. 2012) This produces a solution with a vibrant blue color (See Fig. 1b) where the distinctive color is due to the plasmon resonances of the triangular nanoplates. Samples for the TEM were prepared by simply dipping a holey carbon TEM grid into the solution and placing on filter paper. Separate samples were prepared at intervals of 21, 14, 7 and 3 days and immediately before observation in the TEM. 
Samples were stored in ambient air in an air-conditioned laboratory and no special precautions were taken during storage. The laboratory space contained only the electron microscope and so it is not expected that there is any exposure to fumes of chemical compounds outside of what is typical for ambient air.

\section{Electron Microscopy}

Secondary electron imaging and energy dispersive X-ray spectroscopy (EDS) of the surface of the corroded Ag sheet was performed using a Zeiss Sigma VP FESEM equipped with Bruker light element SSD EDS detector. TEM imaging and EDS spectroscopy on the bulk Ag film were performed in a JEOL $2100 \mathrm{LaB}_{6}$ TEM. High-resolution TEM (HRTEM) lattice imaging of the AgNP was performed in a JEOL2100F TEM.

\section{Results and Discussion}

Fig. 2 shows typical TEM images of the AgNP as they corrode. Within 3 days the formation of smaller secondary particles surrounding the triangles is observed and the triangles start to lose their regular shape. After 7 days the number of surrounding particles has increased and the shape of the triangles is further modified. By 14 days the triangles are almost fully corroded with only the occasional remnants of triangular particles remaining. Some regions of dark contrast are also observed, associated with some Ag that has not been completely converted to $\mathrm{Ag}_{2} \mathrm{~S}$. Even after 21 days small regions of unconverted Ag still remain. The different contrast in the background for 
samples at 0 days arises because an area in which the particles were hanging over the edge of the carbon support film was chosen.

Fig. 3 shows a HRTEM image of a small secondary particle observed after 3 days exposure, taken from the region as indicated by the boxed region in Fig. 2, along with the accompanying fast Fourier transforms (FFTs). Similarly, Fig. 4 shows HRTEM and FFTs for the boxed particles after 7 days exposure. At 3 days, the small newly formed particles are observed to be predominantly finegrained particles having a lattice spacing consistent with pure Ag. At 7 days exposure some of the small particles have started to convert to $\mathrm{Ag}_{2} \mathrm{~S}$, as lattice spacings consistent with of the $\mathrm{Ag}_{2} \mathrm{~S}$ appear, such as the $\{111\},\{-1-12\}$ and $\{120\}$ planes (3.08 $\AA, 2.84 \AA$ and $2.66 \AA$ respectively). The sulfidation is associated with a distinctive dual-contrast appearance of the secondary particles, consisting of a dark area where the lattice spacing is consistent with the Ag fcc lattice and lighter "lobes" that appear amorphous or have lattice spacings consistent with $\mathrm{Ag}_{2} \mathrm{~S}$. The presence of S in the corroded AgNP has also been confirmed with EDS.

The flat triangular AgNP have a distinctive $2.5 \AA$ spacing which has been associated with a $1 / 3\{422\}$ reflection arising from internal stacking faults (Kirkland et al. 1993). Even as the triangles corrode, their distinctive appearance is not significantly altered. This is illustrated in Fig. 5, which is a composite image showing a corroded triangle after 7 days exposure accompanied by smaller very fine-grained polycrystalline Ag particles (as indicated by the Ag $\{111\}$ ring in the FFT). Some of 
these are partially converted to $\mathrm{Ag}_{2} \mathrm{~S}$ and the FFT reveals spots that have lattice spacings that are consistent with $\mathrm{Ag}_{2} \mathrm{~S}$ and are larger than the largest possible lattice spacing in fcc Ag.

At 14 and 21 days exposure, the observed lattice spacings of the particles are predominately from $\mathrm{Ag}_{2} \mathrm{~S}$, but small kernels of polycrystalline Ag still remain. Fig. 6 shows an example of a corroded triangle after twenty-one days exposure, where the original triangular shape can still be distinguished and small amounts of unconverted Ag remain.

Fig. 7a-c show TEM images of the cross-section of the corrosion layer from different areas of the bulk Ag sample. This is the first time, to our knowledge, that cross-sectional images of the corrosion of bulk Ag has been observed. The layer was on the order of 20-50 nm thick and has an inhomogeneous, particulate nature that is not markedly different than that observed as shell around AgNP in previous work (Chen et al. 2013a; Chen et al. 2013b; Elechiguerra et al. 2005). The particulate nature of the layer is also obvious in the SEM image of Fig. 7d. SEM images taken from different regions of the surface, where the corrosion layer had a different color, were indistinguishable. The EDS spectra taken in the SEM showed only the presence of Ag and S. The regions where the corrosion layer was darker had a higher relative S concentration, indicating the corrosion layer was thicker at that point, as expected. Fig. 7e shows an annular dark field image acquired in scanning TEM mode. The brightness is related to the average atomic number of the materials and so the bulk Ag corresponds to the bright regions, the medium grey to the $\mathrm{Ag}_{2} \mathrm{~S}$ 
corrosion layer and the dark areas to the sample adhesive. The corresponding map of the intensity of S peak in the EDS spectrum shown in Fig. 7f confirms this interpretation.

There are also much smaller small particles of around $\sim 5 \mathrm{~nm}$ in size observable in some of the TEM images of the bulk sample, including on the sample adhesive. It is quite likely that these are an artefact produced by redeposition during the ion milling process and it would not be justified at this stage to interpret these as secondary particles formed during the bulk corrosion process. However, the clear particulate nature of the corrosion layer raises the possibility that the corrosion of bulk Ag may also proceed by the dissolution and reprecipitation of secondary Ag particles that are then later converted to $\mathrm{Ag}_{2} \mathrm{~S}$. Further work is required to clarify the role, if any, of the formation of small secondary particles in the corrosion of bulk Ag. This work has demonstrated the potential of crosssectional TEM to examine the corrosion process of bulk Ag and in future work we propose to turn our attention to examine the early stages of the corrosion process. However, there is some indication here that the corrosion process for bulk Ag is not fundamentally different from the AgNP.

The ubiquitous presence of stacking faults in the triangular AgNP may have an important role in both their corrosion and their antibacterial effects. The particles were observed to predominantly corrode from the edge inwards with the release of $\mathrm{Ag}^{+}$ions which migrate away from the triangle and reprecipitate as small polycrystalline particles. The presence of the stacking fault defects intersecting with the surface at the triangle edges may accelerate the ion release at these locations. 
The accelerated ion release when defects intersect the surface can also explain the accelerated corrosion previously observed at the tips of nanorods, as these particles can have a multi-twinned internal structure with defects running along the length of the rod and intersecting at the surface of the tip (Chen et al. 2015; Chen et al. 2013a; Elechiguerra et al. 2005).

The effect of capping agents on the Ag $\{111\}$ surface has been described as accounting for the different corrosion rates for different parts of the AgNP (Chen et al. 2013b). However, $\mathrm{Ag}^{+}$ions have been observed to be able to migrate through thick carbon coatings (Bokhonov 2014) whereas the high coordination number of the Ag $\{111\}$ surface may slow the ion release (Allpress and Sanders 1964). The small polycrystalline secondary nanoparticles are converted to $\mathrm{Ag}_{2} \mathrm{~S}$ much more quickly than the parent AgNP under the exposure conditions used here and this can be attributed to their highly defected structure.

In this work the AgNP have been deposited on an amorphous carbon substrate of the TEM grid and this is likely to play an important role in the accelerated corrosion in comparison to bulk Ag. The presence of a microscopic layer of $\mathrm{H}_{2} \mathrm{O}$ on the surface of the substrate provides a medium in which the $\mathrm{Ag}^{+}$ions can migrate away from the particles, allowing for further release and migration. It is not expected that the nature of the substrate would make a substantial difference to this process unless it was particularly hydrophobic. In the case of bulk Ag, in the absence of a migration pathway, the released ions will remain in an environment of high $\mathrm{Ag}^{+}$concentration which is likely to slow the release of further ions. For the same reasons, when suspended in an aqueous 
environment, very rapid dissolution of the original AgNP will occur. In that case the final destination of the released ions will depend critically on the chemistry of the solution.

\section{Conclusions}

In this work we have demonstrated that there are three (not two as previously discussed) possible mechanisms that must be considered for the corrosion of AgNP and possibly bulk Ag:

(1) direct conversion to $\mathrm{Ag}_{2} \mathrm{~S}$.

(2) oxidative dissolution of $\mathrm{Ag}$ to $\mathrm{Ag}^{+}$followed by precipitation as $\mathrm{Ag}_{2} \mathrm{~S}$.

(3) oxidative dissolution of $\mathrm{Ag}$ to $\mathrm{Ag}^{+}$followed by precipitation as nanoparticulate $\mathrm{Ag}$, then followed by conversion to $\mathrm{Ag}_{2} \mathrm{~S}$.

Which of these processes dominate will be determined by the local environment. It is possible that some of the previous observations in the literature of the formation of $\mathrm{Ag}_{2} \mathrm{~S}$ particles were preceded by the formation of the Ag particles that then subsequently transformed to $\mathrm{Ag}_{2} \mathrm{~S}$ before observation (Chen et al. 2013a; Chen et al. 2013b; Elechiguerra et al. 2005; Lu et al. 2015; Wang et al. 2011). In addition to the presence of water, oxidising species, and the concentration of available $\mathrm{S}$ ions, the exposure to light will also be significant in determining which corrosion process will dominate. The photoinduced conversion of Ag nanoparticles into small clusters by the interaction with light is well-established (Jin et al. 2001). The release of $\mathrm{Ag}^{+}$ions, the availability of a substrate on which they can migrate away from the parent Ag-NP, and their reprecipitation and transformation to $\mathrm{Ag}_{2} \mathrm{~S}$ are all important factors controlling their corrosion and antimicrobial properties. The results 
presented here demonstrate that, despite considerable work to date, it is clear that many questions

about the corrosion of Ag, in both nanoparticle and bulk form, are yet to be resolved.

\section{References}

Alexander JW (2009) History of the medical use of silver Surgical Infections 10:289-292

Allpress JG, Sanders JV (1964) The influence of surface structure on a tarnishing reaction Phil Mag 10:829-836

Andrieux-Ledier A, Tremblay B, Courty A (2013) Stability of self-ordered thiol-coated silver nanoparticles: oxidative environment effects Langmuir 29:13140-13145

Bennett HE, Peck RL, Burge DK, Bennett JM (1969) Formation and growth of tarnish on evaporated silver films J Appl Phys 40:3351-3360

Bokhonov BB (2014) Permeability of carbon shells during sulfidation of encapsulated silver nanoparticles Carbon 67:572-577

Burge DK, Bennett JM, Peck RL, Bennett HE (1969) Growth of surface films on silver Surface Science 16:303-320

Cao W, Elsayed-Ali HE (2009) Stability of Ag nanoparticles fabricated by electron beam lithography Materials Letters 63:2263-2266

Chen R, Nuhfer NT, Moussa L, Morris HR, Whitmore PM (2008) Silver sulfide nanoparticle assembly obtained by reacting assembled silver nanoparticle template with hydrogen sulfide gas Nanotechnology 19:455604

Chen S et al. (2015) Avoiding artefacts during electron microscopy of silver nanomaterials exposed to biological environments J Microsc

Chen S et al. (2013a) Sulfidation of silver nanowires inside human alveolar epithelial cells: a potential detoxification mechanism Nanoscale 5:9839-9847 
Chen S et al. (2013b) High-resolution analytical electron microscopy reveals cell culture mediainduced changes to the chemistry of silver nanowires Environ Sci Technol 47:13813-13821

Chernousova S, Epple M (2013) Silver as antibacterial agent: ion, nanoparticle and metal Angew Chem Int Ed 52:1636-1653

Davidson RA, Anderson DS, Van Winkle LS, Pinkerton KE, Guo T (2014) Evolution of silver nanoparticles in the rat lung investigated by X-ray absorption spectroscopy J Phys Chem A $119: 281-289$

Elechiguerra JL, Larios-Lopez L, Lui C, Garcia-Gutierrez D, Camacho-Bragado A, Yacaman MJ (2005) Corrosion at the Nanoscale: The Case of Silver Nanowires and Nanoparticles Chem Mater 17:6042-6052

Fan M, Brolo AG (2009) Silver nanoparticles self assembly as SERS substrates with near single molecule detection limit Phys Chem Chem Phys 11:7381-7389

Fletcher G, Arnold MD, Pedersen T, Keast VJ, Cortie MB (2015) Multipolar and dark mode plasmon resonances on drilled silver nano-triangles Optics Express 23:18002-18013

Franey JP, Kammlott GW, Graedel TE (1985) The corrosion of silver by atmospheric sulfur gases Corrosion Science 25:133-143

Glover RD, Miller JM, Hutchinson JE (2011) Generation of metal nanoparticles from silver and copper objects: nanoparticle dynamics on surfaces and potential sources of nanoparticles in the environment ACS Nano 5:8950-8957

Huang H, Li Q, Wang J, Li Z, Yu XF, Chu PK (2014) Sensitive and robust colorimetric sensing of sulfide anion by plasmonic nanosensors based on quick crystal growth Plasmonics 9:11-16

Jin R, Cao YW, Mirkin CA, Kelly KL, Schatz GC, Zheng JG (2001) Photinduced conversion of silver nanospheres to nanoprisms Science 294:1901-1903

Keast VJ, Walhout CJ, Pedersen T, Shahcheraghi N, Cortie MB, Mitchell DRG (2016) Higher order plasmonic modes excited in Ag triangular nanoplates by an electron beam Plasmonics doi:DOI 10.1007/s11468-015-0145-6 
Kelly RL, Coronado E, Zhao LL, Schatz GC (2003) The optical properties of metal nanoparticles: the influence of size shape and dielectric environment J Phys Chem B 107:668-677

Kinkhabwala A, Yu Z, Fan S, Avlasevich Y, Mullen K, Moerner WE (2009) Large single-molecule fluorescence enhancements produced by a bowtie nanoantenna Nature Photonics 3:654-657

Kirkland AI, Jefferson DA, Duff DG, Edwards PP, Gameson I, Johnson FG, Smith DJ (1993) Structural studies of trigonal lamellar particles of gold and silver Proc R Soc Lond A 440:589-609

Kleber C, Wiesinger R, Schnöller J, Hilfrich U, Hutter H, Schreiner M (2008) Initial oxidation of silver surfaces by $\mathrm{S}^{2-}$ and $\mathrm{S}^{4+}$ species. Corrosion Science 50:1112-1121

Le Ouay B, Sellacci F (2015) Antibacterial activity of silver nanoparticles: a surface science insight Nano Today 10:339-354

Levard C, Hotze EM, Lowry GV, Brown GE (2012) Environmental transformations of silver nanoparticles: impact of stability and toxicity Environ Sci Technol 46:6900-6914

Levard C, Reinsch BC, Michel FM, Oumahi C, Lowry GV, Jr GEB (2011) Sulfidation processes of PVP-coated silver nanoparticles in aqueous solution: impact on dissolution rate Environ Sci Technol 45:5260-5266

Liu B, Ma Z (2011) Synthesis of Ag2S-Ag nanoprisms and their use as DNA hybridization probes Small 7:1587-1592

Liu J, Pennell KG, Hurt RH (2011) Kinetics and mechanisms of nanosilver oxysulfidation Environ Sci Technol 45:7345-7353

Lu W, Yao K, Wang J, Yuan J (2015) Ionic liquids-water interfacial preparation of triangule Ag nanoplates and their shape-dependent antibacterial activity Journal of Colloid and Interface Science 437:35-41

Marambio-Jones C, Hoek EMV (2010) A review of the antibacterial effects of silver nanomaterials and potential implications for human health and the environment J Nanopart Res 12:15311551 
Marx DE, Barillo DJ (2014) Silver in medicine: the basic science Burns 405:S9-S18

Mayousse C, Celle C, Fraczkiewicz A, Simonato JP (2015) Stability of silver nanowire based electrodes under environmental and electrical stresses Nanoscale 7:2107-2115

McMahon MD, Lopez R, Meyer HM, Feldmen LC, Jr RFH (2005) Rapid tarnishing of silver nanoparticles in ambient laboratory air Appl Phys B 80:915-921

McQueen RH, Keelan M, Xu Y, Mah T (2013) In vivo assessment of odour retention in an antimicrobial silver chloride-treated polyester textile The Journal of the Textile Institute 104:108-117

Miwa M, Watanabe Y (1947) On the structure of silver sulphide and silver-arsenic alloy grown on silver crystals Journal of the Physical Society of Japan 3:52-56

Munusamy P et al. (2015) Comparison of $20 \mathrm{~nm}$ silver nanoparticles synthesized with and without a gold core: structure, dissolution in cell culture media and biological impact on macrophages Biointerphases 10:031003

Pal S, Tak YK, Song JM (2007) Does the antibacterials activity of silver nanoparticles depend on the shape of the nanoparticle? A study of the gram-negative bacterium Escherichia coli Applied and Environmental Microbiology 73:1712-1720

Park G, Lee C, Seo D, Song H (2012) Full-color tuning of surface plasmon resonance by compositional variation of Ay@Ag core-shell nanocubes with sulfides Langmuir 28:90039009

Pastoriza-Santos I, Liz-Marzan LM (2008) Colloidal silver nanoplates. State of the art and future challenges J Mater Chem 18:1724-1737

Philips VA (1962) Role of defects in evaporated silver films on the nucleation of sulfide "patches" J App Phys 33:712-717

Pope D, Gibbens HR, Moss RL (1968) The tarnishing of Ag at naturally-occurring $\mathrm{H}_{2} \mathrm{~S}$ and $\mathrm{SO}_{2}$ levels. Corrosion Science 8:883-887 
Reidy B, Haase A, Luch A, Dawson KA, Lynch I (2013) Mechanisms of silver nanoparticle release, transformation and toxicity: a critical review of current knowledge and recommendations for future studies and applications Materials 6:2295-2350

Reinsch BC et al. (2012) Sulfidation of silver nanoparticles decreases Echerichia coli growth inhibition Environ Sci Technol 46:6992-7000

Rizzello L, Pompa PP (2014) Nanosilver-based antibacterial drugs and devices: Mechanisms, methodological drawbacks, and guidelines Chem Soc Rev 43:1501-1518

Rycenga M et al. (2011) Controlling the synthesis and assembly of silver nanostructures for plasmonic applications Chemical Reviews 111:3699-3712

Shiojiri M, Mada S, Murata Y (1969) Electron microscopy observation of sulphuration of vacuumdeposited silver films Japan J Appl Phys 8:24-31

Sinclair JD (1982) Tarnishing of solver by organic sulfur vapours: rates and film characteristics Electrochemical Science and Technology 239:33-40

Storm-Versloot MN, Vos CG, Ubbink DT, Vermeulen H (2010) Topical silver for preventing wound infection Cochrane Database of Systematic Reviews 3:CD0006478

Thalman B, Voegelin A, Sinnet B, Morgenroth E, Kaegi R (2014) Sulfidation of silver nanoparticles reacted with metal sulfides Environ Sci Technol 48:4885-4892

Theodorou IG et al. (2015) Static and dynamic microscopy of the chemical stability and aggregation state of silver nanowires in components of Murine pulmonary surfactant Environ Sci Technol 49:8048-8056

Toy LW, Macera L (2011) Evidence-based review of silver dressing use on chronic wounds Journal of the American Academy of Nurse Practitioners 23:183-192

Tsuji M et al. (2012) Rapid transformation from spherical nanoparticles, nanorods, cubes, or bipyramids to triangular prisms of silver with PVP, citrate and $\mathrm{H}_{2} \mathrm{O}_{2}$ Langmuir 28:88458861 
Walter N, McQueen RH, Keelan M (2014) In vivo assessment of antimicrobial treated textiles on skin microflora International Journal of Clothing Science and Technology 26:330-342

Wang L et al. (2011) Spectral properties and mechanism of instability of nanoengineered silver blocks Optics Express 19:10640-10646

Xiu ZM, Zhang QB, Puppala HL, Colvin VL, Alvarez PJJ (2012) Negligible particle-specific antibacterial activity of silver nanoparticles Nano Letters 12:4271-4275

Yang P, Portalès H, Pileni M-P (2009) Identification of multipolar surface plasmon resonances in triangular silver nanoprisms with very high aspect ratios using the DDA method J Phys Chem C 113:11597-11604

Zeng J, Tao J, Su D, Zhu Y, Qin D, Xia Y (2011) Selective sulfuration at the corner sites of silver nanocrystal and it's use in stabilization of the shape Nano Letters 11:3010-3015 




Fig. 1. (a) Photograph of a corroded Ag sheet. Circular areas where sections were removed for previous (unrelated) studies can be seen. The box indicates the region of the sheet selected for study here. (b) Photograph of the solution containing flat triangular AgNP. The brilliant blue colour arises from the excitation of plasmon resonances. 


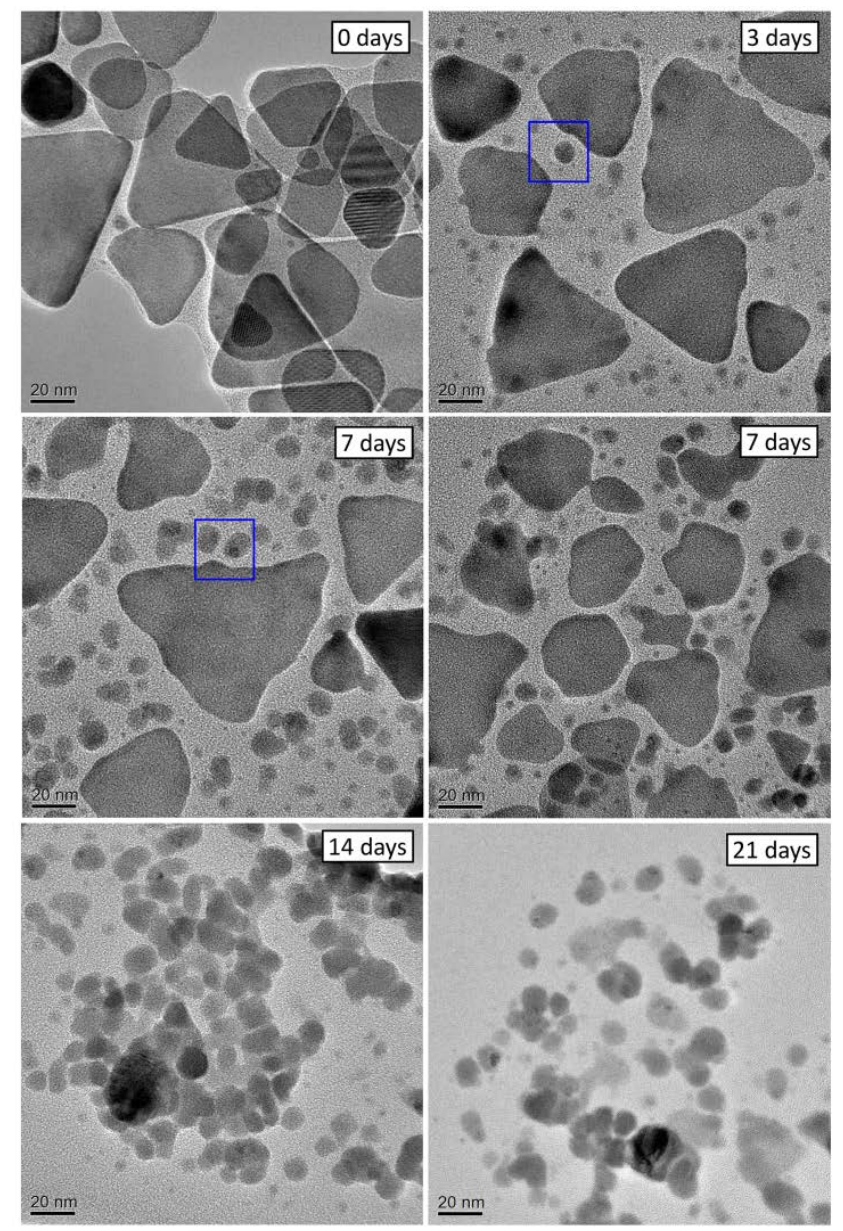

Fig. 2. TEM images of the corrosion of flat triangular AgNP for different lengths of time exposed to ambient laboratory air. HRTEM images of the boxed regions are given in Figs. 3 and 4. 




Fig. 3. (a) HRTEM image of secondary particles formed near a flat triangular Ag-NP after 3 days of exposure to laboratory air. The image corresponds to the boxed region of Figure 2. (b) The FFT of part of the triangle reveals spots associated with the $1 / 3\{422\}$ reflection that are characteristic of triangular nanoplates. (c) The FFT of the smaller particle shows spots corresponding to the $\{111\}$, $\{002\}$ and $\{220\}$ planes of Ag. 


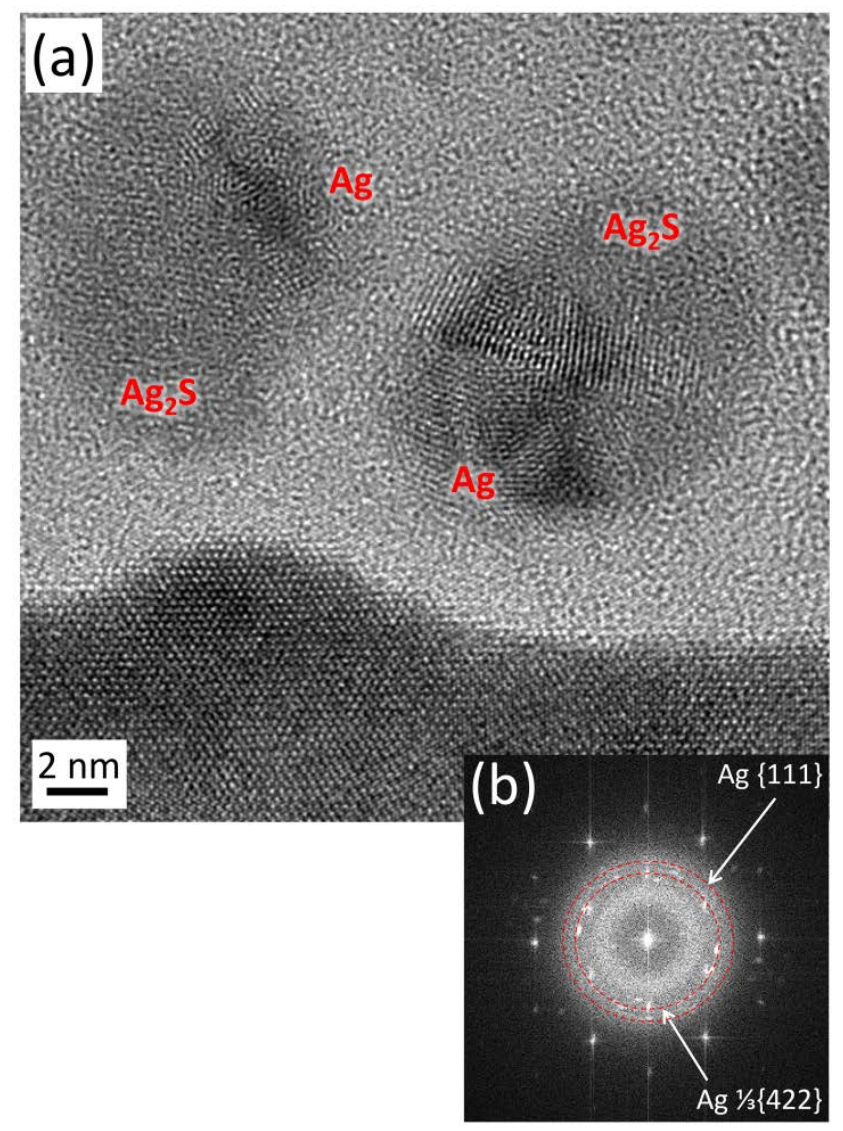

Fig. 4. (a) HRTEM image of secondary particles formed near a flat triangular Ag-NP after 7 days of exposure to laboratory air. The image corresponds to the boxed region of Figure 2. (b) The FFT of the entire region reveals spots associated with the $1 / 3\{422\}$ reflection that are characteristic of triangular nanoplates, spots associated with the $\{111\}$ planes of Ag and larger lattice spacings that can be identified as coming from regions of $\mathrm{Ag}_{2} \mathrm{~S}$. 




Fig. 5. (a) HRTEM image of secondary particles formed near a flat triangular Ag-NP after 7 days of exposure to laboratory air. (b) The FFT of the smaller particles shows a ring corresponding to the $\{111\}$ planes of Ag as well as additional spots corresponding to the larger spacings of $\mathrm{Ag}_{2} \mathrm{~S}$. (c) The FFT of the corroded triangle shows that the characteristic $1 / 3\{422\}$ reflections are unchanged. 


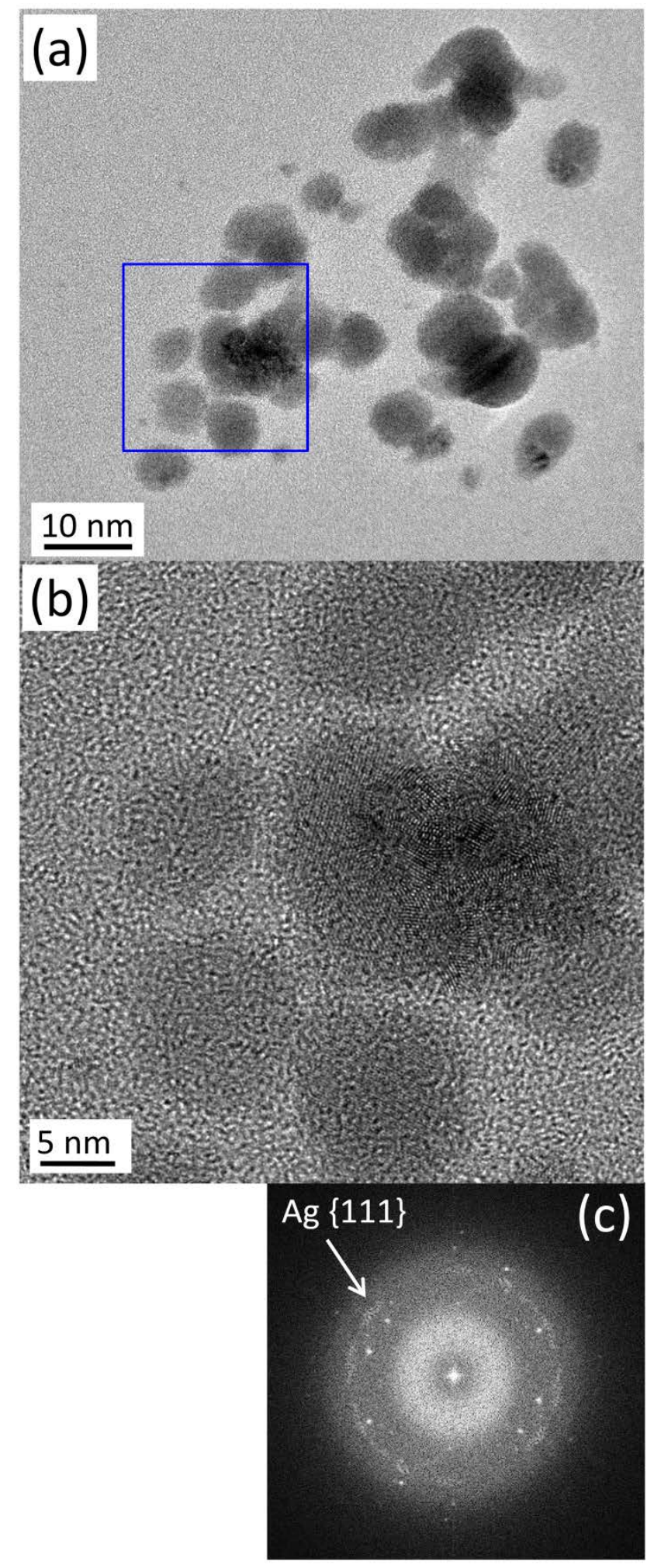

Fig. 6. (a) TEM image of the remnants of a flat triangular Ag-NP after 21 days of exposure to

laboratory air. (b) The HRTEM image shows a kernel of remaining fine-grained polycrystalline Ag with the remainder fully converted to $\mathrm{Ag}_{2} \mathrm{~S}$. (c) The FFT shows a ring corresponding to the $\{111\}$ planes of Ag as well as additional spots corresponding to the larger spacings of $\mathrm{Ag}_{2} \mathrm{~S}$. 

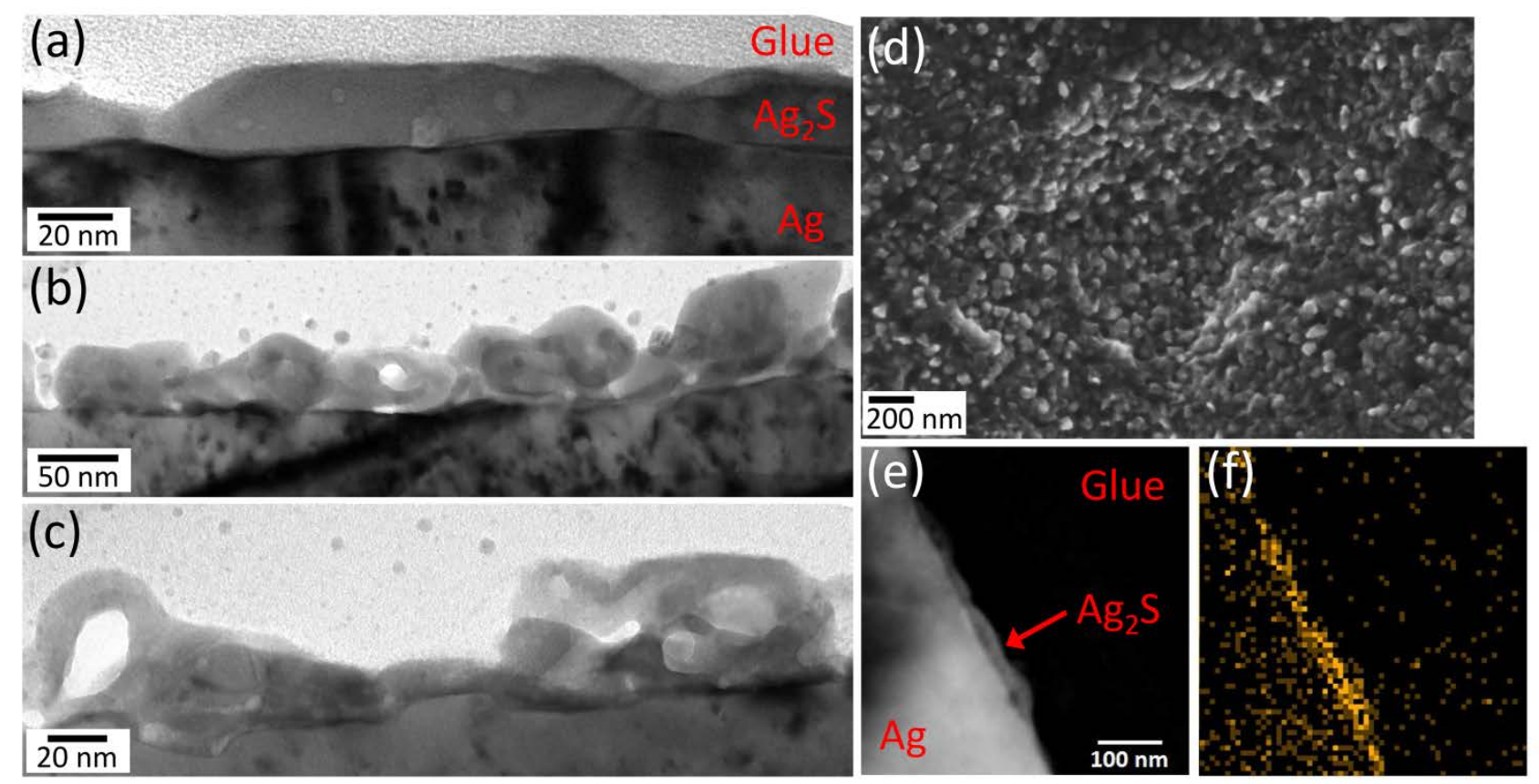

Fig. 7. (a)-(c) TEM cross-sectional images of the corrosion layer formed on a bulk Ag sample after over 10 years exposure to ambient indoor environments. (d) SEM image of the surface of the corrosion layer. (e) Annular dark field image of the corrosion layer (medium gray area) with (f) corresponding EDS map of the S content. 